\title{
Gelenksonografie im Treat-to-Target-Konzept bei der juvenilen idiopathischen Arthritis
}

\section{Musculoskeletal ultrasound in the treat-to-target management of juvenile idiopathic arthritis}

\author{
Autoren \\ Faekah Gohar, Daniel Windschall \\ Institut \\ Klinik für Kinder- und Jugendrheumatologie, St. Josef-Stift \\ Sendenhorst \\ Schlüsselwörter \\ Kinderrheumatologie, Treat-to-Target, Gelenkultraschall, \\ Biomarker, Remissionskriterien \\ Keywords \\ Paediatric rheumatology, treat-to-target, musculoskeletal \\ ultrasound, biomarker, remission criteria \\ Bibliografie \\ DOI https://doi.org/10.1055/a-1341-6971 \\ arthritis + rheuma 2021; 41: 47-52 \\ (c) 2021. Thieme. All rights reserved. \\ Georg Thieme Verlag KG, Rüdigerstraße 14, \\ 70469 Stuttgart, Germany \\ ISSN 0176-5167 \\ Korrespondenzadresse \\ Priv.-Doz. Dr. Daniel Windschall \\ Klinik für Kinder- und Jugendrheumatologie \\ St. Josef-Stift Sendenhorst \\ Westtor 7, 48324 Sendenhorst \\ Deutschland \\ danwindschall@web.de
}

\section{ZUSAMMENFASSUNG}

In unserem Artikel werden aktuelle Bedeutung und Entwicklung der Gelenksonografie als fester Bestandteil eines „Treat-to-Target“-Konzeptes bei der Behandlung der juvenilen idiopathischen Arthritis (JIA) dargestellt und diskutiert. Dabei nimmt die Gelenksonografie neben der klinischen Untersuchung eine entscheidende Rolle in der frühen Diagnosestellung einer JIA ein mit dem Ziel, dem Patienten so früh wie möglich eine effiziente Therapie zukommen zu lassen (Window of Opportunity). Zusätzlich kann die Gelenksonografie, eingebettet in das „Treat-to-Target“-Konzept, eine wichtige Rolle in der Verlaufskontrolle und Evaluation einer subklinischen Entzündungsaktivität spielen. Bisherige etablierte Diagnose-, Verlaufs- und auch Remissionskriterien einer JIA lassen stringente und objektivierbare bildgebende Kriterien vermissen, obwohl sich Qualität und Erfahrung bei der Gelenksonografie stetig verbessern. Neben den sonografischen Kriterien haben sich inzwischen auch verschiedene Biomarker zur Beurteilung der Krankheitsaktivität etabliert. Mit der Entwicklung der Sonografie und sicheren Testung von Biomarkern könnte sich in Zukunft zur frühen Diagnosestellung und sicheren Remissionseinschätzung eine Kombination aus immunologischen und sonografischen Kriterien etablieren, die das bisherige „Treat-to-Target“-Konzept bei der JIA unterstützen und verbessern.

\section{ABSTRACT}

In this article the current role and development of musculoskeletal ultrasound as a central component of treat-to-target management of JIA is discussed. Musculoskeletal ultrasound alongside clinical examination plays a significant role in the early diagnosis of JIA, with the aim of initiating treatment as soon as possible and within the "window of opportunity". Including ultrasound in treat-to-target strategies could play an important role in the monitoring and evaluation of clinical as well as subclinical inflammation. However, despite continuous improvements in the quality and experience of ultrasound capabilities, existing diagnostic, prognostic and remission criteria are still lacking objective imaging-specific criteria. Other than ultrasound, immunological biomarkers have also shown utility for the evaluation of clinical activity. The development of ultrasound and routine testing of biomarkers, and potentially both combined, could allow earlier diagnosis and more accurate prediction of disease remission, thereby supporting and improving the ability to treat JIA to target.
Im letzten Jahrzehnt hat sich der muskuloskelettale Ultraschall (MSUS) zu einer der wichtigsten technischen Untersuchungsmethoden für die Gelenkbeurteilung bei Patienten mit juveniler idiopathischer Arthritis (JIA) entwickelt. MSUS, von Kindern als nicht- invasive bildgebende Methode hervorragend toleriert, ermöglicht die dynamische und zeitäquivalente Darstellung von Gelenkergüssen, synovialer Hyperplasie, Strukturanomalien der Knorpel- und Knochenoberfläche sowie der Vaskularisation der hyperplastischen 

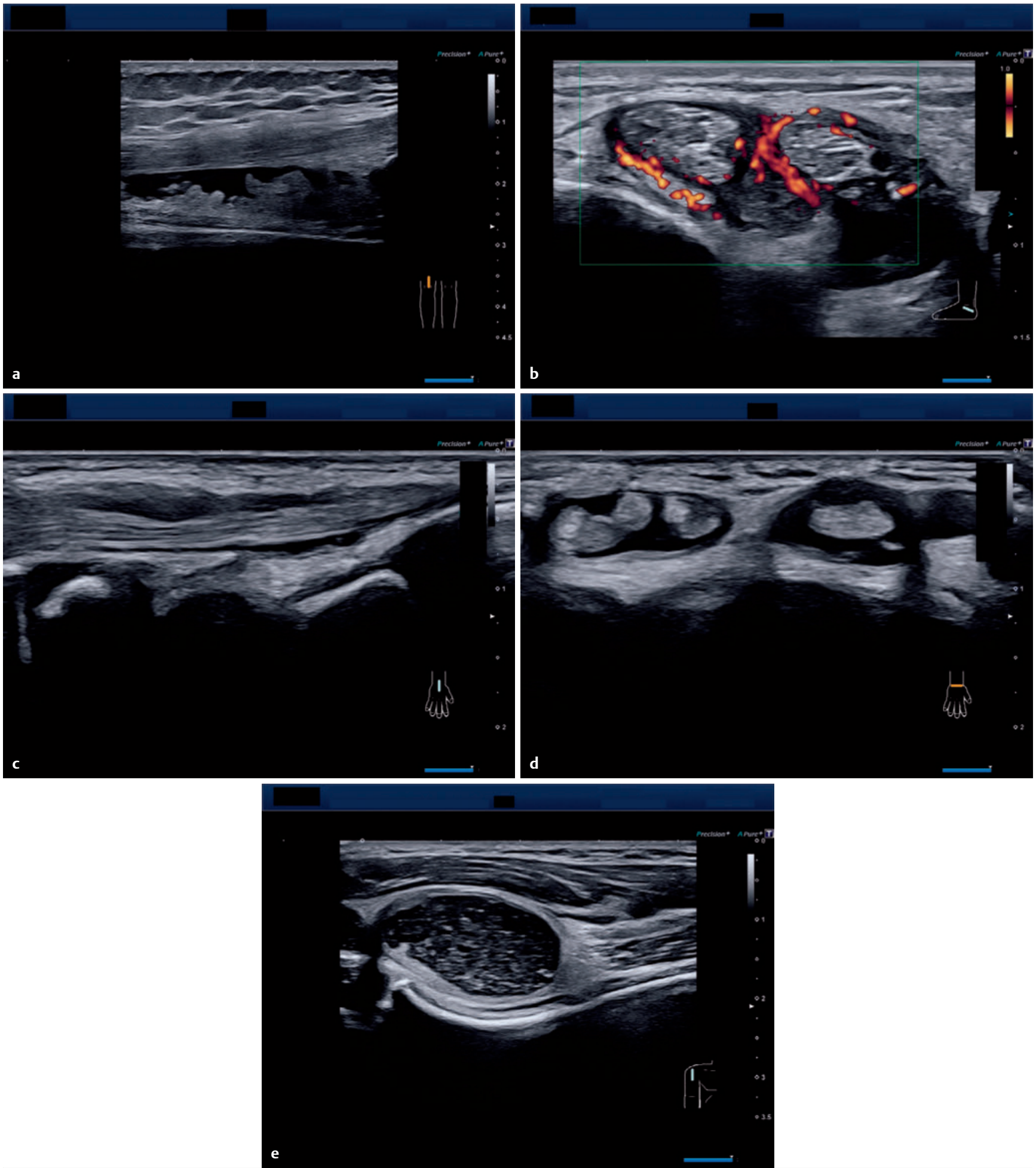

- Abb. 1 Hochauflösender Ultraschall bei Patienten mit JIA: (a) Arthritis des Kniegelenkes mit Erguss und Synovialitis; (b) Tenosynovialitis mit hoher Power-Doppler-Aktivität; (c) Strecksehnenentzündung, Longitudinalschnitt Handgelenk; (d) Strecksehnenentzündung, Transversalschnitt Handgelenk; (e) Synovialzyste, Longitudinalschnitt Bizepssehne/Schulter.

Synovialis. Die Entwicklung einer immer hochauflösenderen Bildgebung ermöglicht die sichere und genaue Evaluation aller Gelenkstrukturen einschließlich der Sehnen. Somit können Arthritis, Tenosynovialitis und Enthesitis genau differenziert werden ( $\triangleright$ Abb. 1). Im Vergleich zum alleinigen Einsatz der klinischen Untersuchung, kann der MSUS die Diagnosestellung einer Arthritis und Tenosynovialitis wesentlich verbessern. Diese Entwicklung führt nicht nur zu einer sicheren Bestätigung einer Arthritis im Frühstadium der JIA, sie ermöglicht auch den sicheren Ausschluss einer entzündlich rheumatischen Erkrankung. Eine häufige Fragestellung in der tägli- 
chen Routine eines Kinderrheumatologen ist die Abgrenzung von Arthralgien und Arthritis, z. B. im Rahmen von sogenannten Wachstumsschmerzen, chronischen Schmerzsyndromen oder Hypermobilität. Die Konsequenzen für die nachfolgende Therapie sind erheblich und Verdachtsmomente sollten hierbei möglichst eindeutig geklärt werden.

Alle Subtypen der JIA sind gekennzeichnet von einer chronischen Entzündung der Synovialis. Wenn diese inadäquat behandelt wird, führt sie zu Knorpelläsionen und Knochenschädigungen mit möglichen Langzeitfolgen wie Funktionsbeeinträchtigungen, Fehlstellungen und Kontrakturen. Nicht nur für die Diagnosestellung findet die moderne Gelenksonografie Anwendung, sondern auch für die präzise Beurteilung der Krankheitsaktivität. Auf diese Weise kann der MSUS zu einer sensitiveren Einschätzung des Therapieerfolges führen und potenziell das Risiko von Krankheitsschüben erfassen. Im klinischen Alltag verlangt der Einsatz der Gelenksonografie zur Beurteilung der Gelenksituation festgelegte Normwerte und Standards, um den Untersuchungsablauf, die sichere Einschätzung der Entzündungsaktivität und den Gelenkschaden zu definieren. Eine große Herausforderung in der Kinderrheumatologie sind die im Vergleich zum Erwachsenen alters- und entwicklungsbedingten Veränderungen des heranreifenden Bewegungsapparates bei Kindern und Jugendlichen. Standards müssen alters- und entwicklungsbezogen definiert und berücksichtigt werden, was in den letzten Jahren durch mehrere Publikationen über die kindliche Sonografie verschiedener Gelenkregionen erleichtert wurde ( Abb. 2) [1-5].

Die optimale Versorgung eines Patienten mit JIA wäre eine frühe und genaue Diagnosestellung gefolgt von einem multidisziplinären Therapieplan mit einem festgelegten Therapieziel (Kriterien für inaktive Erkrankung): das sogenannte „Treat-to-Target“-Konzept. In der internistischen Rheumatologie hat sich das „Treat-to-Target“-Konzept bewährt und zu einer verbesserten Effektivität der Therapie durch eine gezielte Behandlung der Patienten mit medikamentöser Einstellung, Umstellung oder dem Absetzen von Medikamenten nach evidenzbasierten Kriterien geführt. In der Gesellschaft für Kinder- und Jugendrheumatologie befasst sich aktuell eine Kommission „Pro-Kind“ (Protokolle in der Kinderrheumatologie) mit der Entwicklung von Therapieprotokollen für die verschiedenen Kategorien der JIA, die das „Treat-to-Target“-Prinzip verfolgen sollen. Eine flächendeckende Anwendung des Ultraschalls in der pädiatrisch-rheumatologischen Praxis, eine ausreichende Schulung und Expertise sowie konsentierte Standards, stellen notwendige Grundlagen für die Aufnahme der Gelenksonografie in das „Treat-to-Target“-Konzept dar [6].

\section{Behandlungsziele bei der JIA}

Da die JIA eine Ausschlussdiagnose ist und im Kindesalter insgesamt selten auftritt, wird die Diagnose oft erst verzögert gestellt, was zu einer erhöhten Rate an Krankheitskomplikationen führt. Sobald eine JIA diagnostiziert wird, kann durch rasche Einleitung einer adäquaten Therapie die Langzeitprognose wesentlich verbessert werden. Zusätzlich kann eine frühe zielgerichtete Therapie, z. B. mit Biologika, in diesem Window of Opportunity das Outcome verbessern und das Fortschreiten der Erkrankung unterbinden. Patienten müssen in regelmäßigen Intervallen untersucht werden, um die

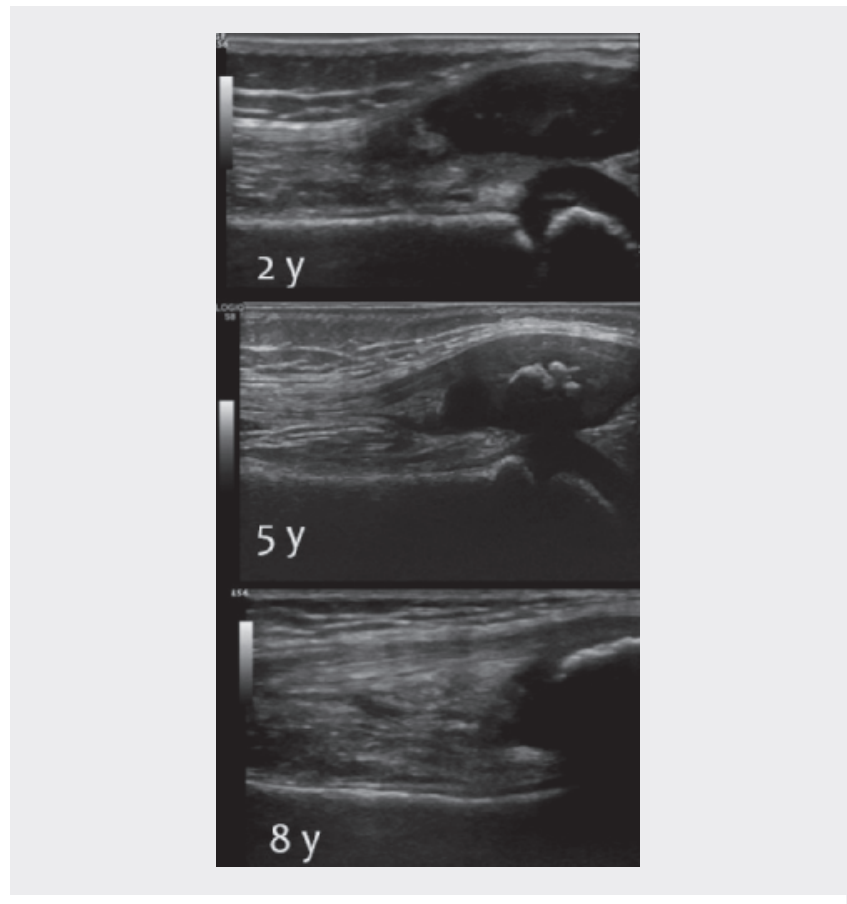

- Abb. 2 Suprapatellarer Longitudinalschnitt in verschiedenen Altersklassen mit zunehmender Ossifikation der Patella.

Fortschritte in Richtung Remission zu evaluieren. Für solche Beurteilungen sind sogenannte Treatment-Targets unabdingbar und benötigen sensitive, diagnostische und quantitative Scoring-Methoden, z. B. mit dem Juvenile Arthritis Disease Activity Score (JADAS). Dieser beinhaltet die Einschätzung der allgemeinen Krankheitsaktivität seitens des behandelnden Arztes als auch des Patienten/der Eltern, die Anzahl der aktiven Gelenke und die normalisierte BSG. In Studien zur JIA wird die klinische Remission oft noch nach den Wallace-Kriterien definiert, welche klinische Parameter, die Messung der BSG und des CRP-Wertes sowie die ärztliche globale Krankheitseinschätzung (PGA, Physician global Assessment) umfassen. Ein Ansprechen auf die Therapie wird oft mit dem ACR-Score („American College of Rheumatology $90 \% / 80 \% / 75 \% / 50 \%$ Criteria for Improvement in JIA Score“) evaluiert [7]. > Abb. 3 fasst die Aufgaben der MSUS für das Erreichen der Behandlungsziele zusammen.

Mehrere Studien konnten bereits nachweisen, dass die Sonografie eine höhere Sensitivität bezüglich einer Gelenkentzündung als die klinische Untersuchung aufweist [8-11]. Zusätzlich können die regelmäßigen MSUS-Untersuchungen auch zur Erkennung von frühen Zeichen einer Gelenkschädigung, wie z. B. unterschiedliches Längenwachstum oder erosive Veränderungen, eingesetzt werden [12]. Diesbezüglich stellt die nicht invasive, relativ kurze und effektive Untersuchung mittels Ultraschall eine äußerst wertvolle diagnostische Methode in der Praxis dar. Mittels MSUS lassen sich die unterschiedlichen betroffenen Strukturen wie die Synovialis, Sehnen und Sehnenansätze genau differenzieren, insbesondere in sehr komplexen Gelenken wie z. B. dem Sprunggelenk [13, 14]. Hierdurch spielt der MSUS eine immer größere Rolle in der Diagnosestellung und Verlaufskontrolle einer JIA [15-17]. 


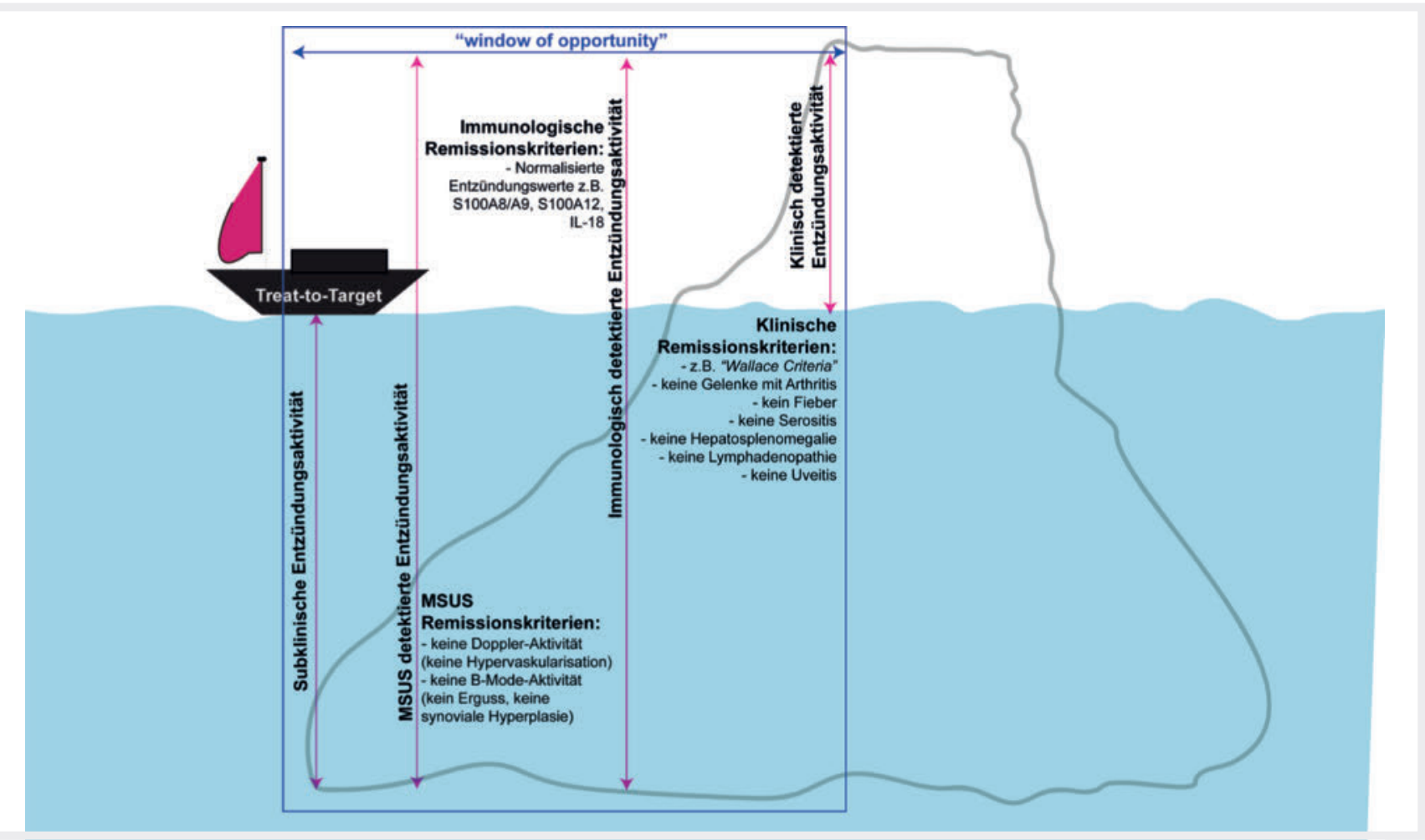

- Abb. 3 Das „Iceberg“-Modell für die subklinische Aktivität bei der JIA modifiziert mit potenziellen Ansprechkriterien und Therapiezielen im „Treat-to-Target“-Behandlungskonzept.

\section{Ultraschallgesteuerte intraartikuläre Glukokortikoidbehandlung}

Der MSUS ist in der Kinderrheumatologie nicht nur zur Diagnosestellung und Verlaufskontrolle bei einer JIA einsetzbar, sondern spielt auch eine wichtige Rolle bei der Nadelführung bei intraartikulären Injektionen. Das Vorgehen beinhaltet die Aspiration von Gelenkergüssen zur Symptomlinderung und Analyse sowie die intraartikuläre Injektion von Glukokortikoiden. Die lokale Behandlung der Gelenke kann sowohl als Brückentherapie bis zum Wirkungseintritt einer medikamentösen Therapie als auch als Initialtherapie z. B. bei Oligoarthritis eingesetzt werden [18]. Das Risiko einer falsch platzierten Injektion sollte insbesondere bei Kindern im Wachstumsalter vermieden werden, um dauerhafte Atrophien des Unterhautfettgewebes sowie Nekrosen zu vermeiden. Mittels Steuerung durch den Ultraschall können kleine Gelenke und Sehnenscheiden besser lokalisiert und angesteuert werden.

\section{Ultraschalldefinierte Krankheitsaktivität}

Zurzeit ist es üblich, den Therapieerfolg einer medikamentösen Therapie bei der JIA 3-4 Monate nach Therapieeinleitung bzw. Dosissteigerung mittels klinischer Aktivität und laborchemischer Entzündungsparameter zu kontrollieren. Mittels MSUS konnte in einer Studie sehr sensitiv der Entzündungsgrad einer Synovialitis bei Kindern bei Neuauftreten/Erstdiagnose der Erkrankung vor Therapiebeginn bestimmt werden [10]. In der gleichen Studie konnte gezeigt werden, dass eine klinische Remission aber nicht mit einer „sonografischen Remission“ gleichgesetzt werden kann, da eine sonografische Aktivität in mehr als der Hälfte der Patienten ohne klinische Symptome nachweisbar war. Somit könnte in Zukunft die sonografische Remission als definiertes Therapieziel einen wichtigen Ansatz für eine bessere „Treat-to-Target“-Strategie bei der JIA bedeuten.

In einer internationalen prospektiven Studie konnte in Biologika-naiven Patienten mittels B-Mode und Doppler eine Synovialitis vor Beginn einer Abatacept-Therapie nachgewiesen werden. Bereits wenige Wochen nach Therapieeinleitung zeigte sich eine Reduktion des Dopplersignals und der durch die Entzündung bedingten Hypervaskularisation. Die Synovialishyperplasie nahm bereits nach der 2 . Woche ab und die Gelenkergüsse ab der 4 . Woche nach Therapiebeginn [12]. Ähnliche Studien sind bei Patienten mit JIA notwendig, sobald die Kriterien und Entzündungs-Scores für eine kindliche Synovialitis evaluiert wurden. Die OMERACT-Gruppe, die sich in der Rheumatologie um Definitionen und Standards auch im Gelenkultraschall sehr bemüht, hat inzwischen auch eine pädiatrische Ultraschall-Arbeitsgruppe. Aktuelle Projekte untersuchen die Präzision und Anwendbarkeit von existierenden Definitionen für Synovialitis, Tenosynovialitis und Enthesitis in der pädiatrischen Population. Eines der aktuellen Hauptziele der pädiatrischen OMERACT-Arbeitsgruppe ist die Graduierung der Synovialitis in großen und kleinen Gelenken bei Kindern. Ein spezifischer Score für das Kniegelenk wurde von der CARRA-Gruppe im vergangenen Jahr publiziert [19]. Offen bleibt die Frage, ob die Untersuchung einer bestimmten Anzahl von definierten Gelenken ausreichend ist, um den Therapieerfolg zu evaluieren. Collado et al. publizierten in einer ersten Studie zu dieser Fragestellung, dass die Berücksichtigung von 10 Gelenken genauso effektiv ist wie die aufwen- 
dige Evaluation von 44 Gelenken, die in der kinderrheumatologischen Routine nur schwer zu bewältigen ist [5].

\section{Ultraschall-Evaluation einer subklinischen Entzündungsaktivität}

Eine subklinische Entzündung ist eine persistierende Krankheitsaktivität ohne sichtbare klinische Krankheitszeichen. Es ist noch nicht eindeutig geklärt, ob eine subklinische Aktivität zu Langzeitschäden führt. Die üblichen im Serum messbaren Entzündungsparameter, wie CRP und BSG, sind nicht immer im Rahmen einer klinisch aktiven JIA erhöht. Aus diesem Grunde sind diese Parameter nicht wirklich brauchbar, um ein Remissionsstadium sicher festzustellen. Erhöhte Akute-Phase-Proteine spielen aber eine größere Rolle bei der systemischen JIA (sJIA), und der Nachweis von z. B. CCP-AK bei seropositiver Polyarthritis weist auf eine schlechtere Prognose hin [20]. Ein systematisches Review hat 15 potenzielle validierte und nicht validierte Entzündungsmarker identifiziert, die für die Prognose einer erhöhten Krankheitsaktivität oder als Unterscheidungsmerkmal zwischen aktiver und inaktiver sJIA-Erkrankung dienen können, aber nur wenige davon wurden in Validierungsstudien getestet [21]. Darunter waren die S100-Proteine S100A8/A9 (MRP8/14) und S100A12, die sowohl diagnostisch als auch als Aktivitätsmarker einsetzbar sind. Patienten mit JIA und persistierend hoher S100-Protein-Konzentration hatten nach dem Absetzen von Methotrexat ein höheres Rezidivrisiko als jene mit normaler Konzentration [22]. Anink et al. berichten, dass eine höhere S100A8/ A9-Konzentration mit einem guten Ansprechen auf eine TNF-alphaTherapie und höhere Spiegel zum Zeitpunkt des Absetzens der Therapie mit einem Rezidiv assoziiert sind [23]. Bei Patienten mit rheumatoider Arthritis (RA), die mit dem TNF-alpha-Blocker Adalimumab behandelt wurden, korrelierte der Rückgang der S100A8/ A9 Serumkonzentration mit einer reduzierten Anzahl an betroffenen Gelenken [24].

Die Gelenksonografie könnte eine wichtige Rolle in der Evaluation einer subklinischen Remission spielen. Es bleibt aber unklar, ob die Behandlung einer subklinischen Synovialitis das Risiko einer leisen Progression mit konsekutiver Gelenkschädigung verringern kann. De Lucia et al. haben eine Kohorte von klinisch inaktiven Patienten evaluiert und konnten zeigen, dass eine sonografische Restsynovialitis signifikant mit einem erhöhten Risiko für einen erneuten Krankheitsschub assoziiert ist [25]. Insbesondere positive Dopplersignale in der vermehrt durchbluteten Synovialis scheinen eine bedeutende Rolle als Hinweis für ein Krankheitsrezidiv zu spielen. Persistierende pathologische Dopplersignale korrelieren mit rekurrierender Synovialisentzündung und der Entwicklung von erosiven Schäden bei der RA [17, 26]. Die klinische Relevanz von hypervaskularisierten Enthesitiden und Sehnenscheiden bei Kindern, die sich ansonsten in klinischer Remission befinden, bleibt offen und bedarf weiterer Untersuchungen.

\section{Ultraschallaktivität korreliert mit Biomarkern im Serum und in der Synovialflüssigkeit}

Die Korrelation von sonografischen Veränderungen mit biochemischen Markern ist ein interessantes Gebiet und könnte die Behandlung der JIA sehr positiv beeinflussen. So könnte es sein, dass bestimmte MSUS-Veränderungen hochsensitiv mit gewissen serologischen Markern, z. B. angiogenetischen Markern und Zytokinen korrelieren, wie bisher bei der RA untersucht wurde. Die Identifizierung eines bestimmten Musters könnte die Therapiewahl optimieren. Ramirez et al. konnten zeigen, dass in einer Kohorte von Patienten in klinischer Remission aber mit noch sonografisch nachweisbarer Synovialitis, $45 \%(n=25)$ der Patienten erhöhte angiogenetische Biomarker ohne Nachweis von proinflammatorischen Zytokinen hatten [27]. Ähnliche Ergebnisse konnten von Misra gezeigt werden: Patienten mit Hypervaskularisation Grad 3 hatten höhere Serumlevel von angiogenetischen Entzündungsmarkern verglichen mit Patienten mit einer milden Power-Doppler-Aktivität Grad 2, obwohl die letztere Gruppe eine hohe klinische Krankheitsaktivität und die erste nur milde Krankheitszeichen aufwies [28]. Die Serum-IL-17-Konzentration korreliert mit Hypervaskularisation in den Kniegelenken bei Früharthritis und weist auf das Potenzial einer frühen Behandlung mit IL-17-Antagonisten hin [29]. Andere Entzündungsmarker, die mit einer sonografisch definierten Synovialitis korrelieren, könnten IL6 oder S100A8/A9 bei Patienten mit RA oder möglichweise auch JIA sein [30, 31]. Aktuell liegen nur wenige Daten zur Korrelation von Biomarkern und Ultraschallbefunden bei der JIA vor [32].

\section{FAZIT}

Die Gelenksonografie ist eine praxisnahe und zunehmend sensitive bildgebende Methode zur Detektion und Lokalisierung von Entzündungen bei der JIA. MSUS zeigt auch eine Sensitivität für subklinische Entzündungen und könnte in Zukunft eine wesentlich größere Rolle zur Beurteilung einer Remission im „Treat-to-Target“-Konzept spielen. Sehr wichtig werden in den nächsten Jahren Studien sein, um definierte MSUS-Kriterien, prinzipiell auch kombiniert mit serologischen Markern, für die JIA zu standardisieren und zu evaluieren. Die Vorteile vom pädiatrischen MSUS im klinischen Alltag sind inzwischen anerkannt. In der Einschätzung und Messung des Therapieansprechens und der Remission werden die Vorzüge der Gelenksonografie aktuell noch vernachlässigt und unterschätzt.

Interessenkonflikt

Die Autorinnen/Autoren geben an, dass kein Interessenkonflikt besteht.

Literatur

[1] Collado P, Naredo E, Calvo C et al. Reduced joint assessment vs comprehensive assessment for ultrasound detection of synovitis in juvenile idiopathic arthritis. Rheumatol (United Kingdom) 2013; 52(8): 1477-1484

[2] Weiss PF, Chauvin NA, Klink AJ et al. Detection of enthesitis in children with Enthesitis-related arthritis: dolorimeter examination compared to ultrasonography. Arthritis Rheumatol 2014; 66(1): 218-227 
[3] Roth J, Ravagnani V, Backhaus M et al. Preliminary Definitions for the Sonographic Features of Synovitis in Children. Arthritis Care Res 2017; 69(8): 1217-1223

[4] Windschall D, Collado P, Vojinovic J. Age-Related Vascularization and Ossification of Joints in Children: An International Pilot Study to Test Multiobserver Ultrasound Reliability. Arthritis Care Res 2020; 72(4): 498-506

[5] Collado P, Vojinovic J, Nieto JC et al. Toward Standardized Musculoskeletal Ultrasound in Pediatric Rheumatology: Normal Age-Related Ultrasound Findings. Arthritis Care Res 2016; 68(3): 348-356

[6] Ravelli A, Consolaro A, Horneff $G$ et al. Treating juvenile idiopathic arthritis to target: recommendations of an international task force. Ann Rheum Dis 2018; 77: 819-828

[7] Vastert SJ, de Jager W, Noordman BJ et al. Effectiveness of First-Line Treatment With Recombinant Interleukin-1 Receptor Antagonist in Steroid-Naive Patients With New-Onset Systemic Juvenile Idiopathic Arthritis: Results of a Prospective Cohort Study. Arthritis Rheumatol [Internet] 2014 Apr [cited 2014 Jun 3]; 66(4): 1034-1043. Available from: http://doi.wiley.com/10.1002/art.38296

[8] Breton S, Jousse-Joulin S, Cangemi C et al. Comparison of Clinical and Ultrasonographic Evaluations for Peripheral Synovitis in Juvenile Idiopathic Arthritis. Semin Arthritis Rheum [Internet] 2011; 41(2): 272-278. Available from: http://www.sciencedirect.com/science/article/pii/S0049017211000023

[9] Rebollo-Polo M, Koujok K, Weisser C et al. Ultrasound findings on patients with juvenile idiopathic arthritis in clinical remission. Arthritis Care Res 2011; 63(7): 1013-1019

[10] Lanni S, van Dijkhuizen EHP, Vanoni F et al. Ultrasound changes in synovial abnormalities induced by treatment in juvenile idiopathic arthritis. Clin Exp Rheumatol [Internet] 2018; 36(2): 329-334. Available from: http://europepmc.org/abstract/MED/29185965

[11] Magni-Manzoni S, Scirè CA, Ravelli A et al. Ultrasound-detected synovial abnormalities are frequent in clinically inactive juvenile idiopathic arthritis, but do not predict a flare of synovitis. Ann Rheum Dis [Internet] 2013 Feb 1; 72(2): 223 LP-228. Available from: http://ard. bmj.com/content/72/2/223.abstract

[12] D’Agostino MA, Wakefield RJ, Berner-Hammer $\mathrm{H}$ et al. Value of ultrasonography as a marker of early response to abatacept in patients with rheumatoid arthritis and an inadequate response to methotrexate: Results from the APPRAISE study. Ann Rheum Dis 2016; 75(10): $1763-1769$

[13] Pascoli L, Wright S, McAllister C, Rooney M. Prospective Evaluation of Clinical and Ultrasound Findings in Ankle Disease in Juvenile Idiopathic Arthritis: Importance of Ankle Ultrasound. J Rheumatol [Internet] 2010 Nov 1; 37(11): 2409 LP-2414. Available from: http://www. jrheum.org/content/37/11/2409.abstract

[14] Rooney ME, McAllister C, Burns JFT. Ankle Disease in Juvenile Idiopathic Arthritis: Ultrasound Findings in Clinically Swollen Ankles. J Rheumatol [Internet] 2009 Aug 1; 36(8): 1725 LP-1729. Available from: http:// www.jrheum.org/content/36/8/1725.abstract

[15] Chauvin NA, Khwaja A. Imaging of Inflammatory Arthritis in Children: Status and Perspectives on the Use of Ultrasound, Radiographs, and Magnetic Resonance Imaging. Rheum Dis Clin North Am 2016; 42(4): 587-606

[16] Malattia C, Tzaribachev N, van den Berg JM, Magni-Manzoni S. Juvenile idiopathic arthritis - the role of imaging from a rheumatologist's perspective. Pediatr Radiol 2018; 48(6): 785-791

[17] Magni-Manzoni S, Malattia C, Lanni S, Ravelli A. Advances and challenges in imaging in juvenile idiopathic arthritis. Nat Rev Rheumatol 2012; 8(6): 329-336

[18] Collado P, Windschall D, Vojinovic ] et al. Amendment of the OMERACT Ultrasound Definitions of Joints' Features in Healthy Children When Using the Doppler Technique. Pediatr Rheumatol Online J 2018; 16(1): 23
[19] Oo W, Linklater J, Bennell K et al. Construct Validity of OMERACT Ultrasound Knee Scores with Pain, Other Symptoms, Radiographic and MRI Findings [abstract]. Arthritis Rheumatol 2019; 71(suppl 10)

[20] Gilliam BE, Chauhan AK, Low JM, Moore TL. Pediatric rheumatology. Measurement of biomarkers in juvenile idiopathic arthritis patients and their significant association with disease severity : a comparative study. Clin Exp Rheumatol [Internet] 2008 Jan [cited 2014 Apr 25]; 26(3): 492-497. Available from: http://www.ncbi.nlm.nih.gov/pubmed/18578976

[21] Gohar F, Kessel C, Lavric M et al. Review of biomarkers in systemic juvenile idiopathic arthritis: helpful tools or just playing tricks? Arthritis Res Ther [Internet] 2016 Jul 13 [cited 2018 Mar 4]; 18: 163. Available from: http://www.ncbi.nlm.nih.gov/pubmed/27411444

[22] Foell D, Wulffraat N, Wedderburn LR et al. Methotrexate withdrawal at 6 vs 12 months in juvenile idiopathic arthritis in remission: a randomized clinical trial. JAMA [Internet] 2010 Apr 7 [cited 2014 Apr 24]; 303(13): 1266-1273. Available from: http://jama.jamanetwork.com/ article. aspx?articleid $=185632$

[23] Anink J, Van Suijlekom-Smit LWA, Otten MH et al. MRP8/14 serum levels as a predictor of response to starting and stopping anti-TNF treatment in juvenile idiopathic arthritis. Arthritis Res Ther [Internet] 2015 Jan [cited 2015 Aug 10]; 17(1): 200. Available from: http://arthritis-research.com/content/17/1/200

[24] Hammer HB, Fagerhol MK, Wien TN, Kvien TK. The soluble biomarker calprotectin (a S100 protein) is associated to ultrasonographic synovitis scores and is sensitive to change in patients with rheumatoid arthritis treated with adalimumab. Arthritis Res Ther [Internet] 2011; 13(5): R178. Available from: http://arthritis-research.com/content/13/5/R178

[25] De Lucia O, Ravagnani V, Pregnolato F et al. Baseline ultrasound examination as possible predictor of relapse in patients affected by juvenile idiopathic arthritis (JIA). Ann Rheum Dis [Internet] 2018 Oct 1; $77(10)$ : 1426 LP-1431. Available from: http://ard.bmj.com/content/77/10/1426.abstract

[26] Nguyen H, Ruyssen-Witrand A, Gandjbakhch F et al. Prevalence of ultrasound-detected residual synovitis and risk of relapse and structural progression in rheumatoid arthritis patients in clinical remission: A systematic review and meta-analysis. Rheumatol (United Kingdom) 2014; 53(11): 1-9

[27] Ramírez ], Ruíz-Esquide V, Pomés I et al. Patients with rheumatoid arthritis in clinical remission and ultrasound-defined active synovitis exhibit higher disease activity and increased serum levels of angiogenic biomarkers. Arthritis Res Ther 2014; 16(1)

[28] Misra S, Mondal S, Chatterjee S et al. Association of Angiogenic and Inflammatory Markers with Power Doppler Ultrasound Vascularity Grade and DAS28-CRP in Early Rheumatoid Arthritis: A Comparative Analysis. Biomed Res Int 2018; 2018

[29] Gullick NJ, Evans HG, Church LD, Jayaraj DM, Filer A, Kirkham BW, et al. Linking power doppler ultrasound to the presence of Th17 cells in the rheumatoid arthritis joint. PLoS One 2010; 5(9): 1-11.

[30] do Prado AD, Bisi MC, Piovesan DM et al. Ultrasound power Doppler synovitis is associated with plasma IL-6 in established rheumatoid arthritis. Cytokine 2016; 83: 27-32

[31] Kang KY, Woo JW, Park SH. S100A8/A9 as a biomarker for synovial inflammation and joint damage in patients with rheumatoid arthritis. Korean J Intern Med 2014; 29(1): 12-19

[32] Świdrowska J, Smolewski P, Stańczyk J, Smolewska E. Serum Angiogenesis Markers and Their Correlation with Ultrasound-Detected Synovitis in Juvenile Idiopathic Arthritis. J Immunol Res 2015; 2015: 741457. doi: 10.1155/2015/741457. Epub 2015 May 4. PMID: 26065004; PMCID: PMC4434192 